\title{
Les Moyens de Communication Chez les Igbo
}

\author{
Samuel Maduagwuna \& Oluchukwu Asadu* \\ http://dx.doi.org/10.4314/ujah.v14i2.7
}

\section{Résumé}

La communication joue des rôles très importants chez les Igbo. Les rôles comprennent entre autres le partage d' information, des nouvelles sentiments aussi bien que le passage d'idée et de message à autrui. Des moyens divers sont déployés pour effectuer la communication. D'abord il y a des organes phonatoires et ensuite les instruments tels que la flute (oja), les gongs métalliques (ogene ou ubom), les gongs en bois (ikoro ou ekwe), le tam-tam (igba) et le xylophone. Ces instruments sont employés pour communiquer surtout pendant les cérémonies. Ce travail a pour but l'exposition de la fonction de ces instruments comme moyens de communication chez les igbo.

\section{L'Introduction}

La communication est un aspect de la vie employée à chaque moment. Elle sert à communiquer des idées, des opinions, des pensées ou des sentiments. Bien que le terme soit employé toujours et des idées soient évoquées chaque fois qu'il est prononcé, il est conçu et défini des manières différentes. Avant de passer à la définition du mot il faut tout d'abord traiter l'étymologie du mot.

Selon certains savants, le mot communication est dérivé du verbes latin communicare qui signifie "parler ensemble", discuter ou consulter l' un et l'autre; et pour d'autres le mot a pour origine le nom latin "communes" ce qui veut dire "commun". La revendication de chaque groupe se 
justifie dans l'argument que la communication implique $<<$ rendre commun un message à tous $>>$. Pour les auteurs de ce travail, l'origine de communication importe peu. L'accent est plutôt sur la signification, le concept ou le sens.

Le but principal de la communication est d'affecter le comportement et la situation du récepteur d'un message.Dans la tentative de la définition de la communication Hoben (1954) dit ${ }^{<C}$ Communication is the verbal interchange of thought or idea. ${ }^{>>}$Version française: La communication est l'échange verbal de pensée ou d'idée. La communication implique la compréhension entre le locuteur et l'interlocuteur. Pour Barnlund (1964) ${ }^{<}$Communication arises out of the need to reduce uncertainty, to act effectively, to defend or strengthen the $\mathrm{ego}^{>>}$.Verson française: $\mathrm{La}$ nécessité de réduire l'incertitude, d'agir effectivement, de defendre ou de fortifier l'égo est à la base de la communication.

A partir de ces définitions on comprend que la communication fait allusion au partage d'information, d'idée, des nouvelles, de sentiment etc, avec les autres tout en rendant commun l'information, le message, l'idée, les nouvelles ou sentiments à tout le monde.

Pour Robert (1985) la communication est ${ }^{\ll}$ la chose que l'on communique ${ }^{\gg>}$. Par exemple "annonce, avis, dépêche, message, note, nouvelle, renseignement". Ce qui est communiqué est à la portée de tous et facilite la vie communautaire. La communication met en lumière la connaissance et la vie clandestine ne peut pas réussir dans la communauté où l'efficacité de la communication est reconnue.

Quels sont les moyens de la communication? Quels sont les moyens de communication chez les igbo? On peut classifier les moyens de communication en deux - les moyens modernes et les moyens primitives.Par les moyens modernes, les personnes communiquent les messages à travers des 
appareils. On peut parler de la communication téléphonique, radiophonique etc.

La communication primitive s'effectue par le biais des organes phonatoires chez l'homme, le gong en bois, le gong métallique, le tam-tam, le tambour in etc.De nos jours, la communication moderne est réalisée très souvent dans les grandes villes et cités en utilisant les hauts parleurs. Mais la communication non technique se réalize jusqu' à présent dans les endroits reculés grace aux instruments comme le tam-tam, le tambourin, les gongs métalliques et les gongs en bois.

Chez les Igbo, il y a plusieurs moyens de communication. Tout d'abord il y a les organs phonatoires. On emploi les organes pour la production des paroles et des hululements.D'autre part, on utilize les instruments comme les gongs de tailles diverses, la flute traditionnelle, le tambourin, le tam-tam etc. On emploi ces instruments pour assurer la communication chez les Igbo. Malgré l'emploi des techniques modernes cette mode de vulgarization d'annonce, d'idée, de nouvelle est toujours en vogue. Ces moyens de communication seront discuté à fond sans oublier la présentation des choses comme la craie blanche et la noix de cola à une visite. Cellesci symbolisent l'accueil chalereux.

\section{Les Modes Diverses de la Communication}

Dans la communication on peut identifier les modes diverses qui dépendent de la modalité. Trios types de communication sont identifiables; à savoir la communication intrapersonnelle, la communication interpersonnelle et la communication de masse.

\section{La Communication Intrapersonnelle}

'Intra'est un élément savant emprunté au latin. Ceci signifie à l'interieur. Ce type de communication qui est fondamental à toute forme de communication humaine implique que 
l'interlocuteur est aussi le locuteur. On parle à soi-même. La communication intrapersonnelle est très importante car sans elle l'être ne peut pas fonctionner dans son environnement. L'être ne sera pas capable de s'ouvrir aux formes externes de la communication. Ce genre de communication implique l'échange de messages entre le cerveau et les organes de sens. A partir de la communication intrapersonnelle on sera en mesure de prendre des decisions fondées sur l'information reçue par le biais du sens. Par exemple, quand on écoute une emission radiophonique surtout la musique, l'oreille est ouverte pour recevoir le son. Si la musique n'est pas intéressante, le cerveau retourne le message à travers le système de communication intrapersonnelle au muscle. La manifestation de manque d'interêt à la musique est soit la fermeture de la radio ou le changement de fréquence.

La communication intrapersonnelle remplit tout le processus connu de la communication. Dans ce processus, c'est le cerveau parmi les autres qui sont les expediteurs du message original par le biais des canaux de communication (le système nerveu central), à un récepteur (le cerveau).

La communication intrapersonnelle est un aspect de la communication utilisée très souvent chez les Igbo. Elle se manifeste la nuit quand on frappe le gong métallique qui précède l'annonce.

\section{La Communication Interpersonnelle}

Dans ce genre de communication, deux ou plus de personnes participent au partage d'idée, d'information, d'opinion, d'expérience etc. La communication se fait face à face ou tête à tête. Le feedback se produit dans l'immédiat et il est donné en forme verbale ou non verbale car les individus qui participent à la communication peuvent utiliser les mots, les mimes ou les gestes pour montrer que le message est bien reçu 
et compris. Ce genre de communication se manifeste dans une pièce de théâtre, dans un dialogue ou dans une conversation. Chez les Igbo, la communication interpersonnelle se voit dans le marché entre un acheteur et un vendeur, au cours de la négotiation pour le marriage traditionnel ou au cours de la réunion au niveau familial.On emploie les organes phonatoires le plus souvent dans une telle situation.

\section{Communication de Masse}

La communication de masse est différente des deux genres qu'on vient de discuter. Dans le système de communication de masse, les messages sont transmis à un grand public ou foule simultanément en utilisant des voies artificiels ou des machines qui s'appellent les médias. Selon Robert (1985), média est "tout support de diffusion massive de l'information (presse, radio, television, cinema, publicité etc)". Merrily et al (1979) les qualifient de voies artificiels puisqu' ils sont établis pour agir de la part des personnes.

\section{Les Genres Traditionnels de Communication Chez Les Igbo}

Les genres traditionnels de communication chez les Igbo sont nombreux. Ils comprennent entre autres les paroles, l'hululement, les mimes, les gestes, la touche, les images, les symbols, la musique et la dance.La communication traditionnelle chez les igbo fait allusion au commandement, aux informations, aux avertissements, aux conseils etc. On peut les transmettre grace au changement de grimace, à l'emploi des peintures, de la musique, de la pièce, etc.

Les genres de communication chez les Igbo seront repartis en deux groupes; à savoir la communication interpersonnelle et la communication de masse. Dans les deux catégories de communication on doit souligner qu'il y a des 
fois la défaillance dans la communication en utilisant ces modes. Dans la communication interpersonnelle on utilise les organes phonatoires et d'autres moyens comme les gestes, les mimes, l'hululement, les symbols, les objets etc.

La communication de masse dans la société traditionnelle amplifie l'emploi des instruments utilisés aussi pour faire la musique.La communication est très indispensable dans la vie social. Elle soit verbale soit non verbale joue des rôles nécessaries dans la vie quotidienne chez les igbo.

\section{La Communication Non-Verbale Chez Les Igbo}

La communication non-verbal chez les Igbo comprend les gestes, les symbole, les objets, etc.Selon Robert (1985) le geste est "le mouvement du corps (principalement des bras, des mains, de la tête) volontaire ou involontaire [...] visant à exprimer". Le geste est employé pour transmettre un message. Dans ce contexte, il est considéré comme une langue car il remplit le rôle de communication. Le geste est employé pour mettre l'emphase sur un point ou pour communiquer dans une situation où il n' y a pas de parole. Dans le dernier cas il est employé par un groupe social ou groupe de pair. Parfois le groupe organise la devinette dans l'enceinte des membres. L'un fait un geste et l'autre fait l'interprétation.Le rôle important du geste est l'enrichissement de la langue. Il facilite aussi la compréhension dans la communication.

Les objets et symboles jouent aussi des rôles dans la communication. Les objets et symboles comprennent la craie blanche la noix de cola, le rameau de palmier.Chez les Igbo, on présente la craie blanche et la noix de cola. Les deux objets expriment l'accueil chalereux reservé à la visite. La visite, pour réciproquer prend la craie blanche et fait des lignes sur le sol. On brise la noix de cola en faisant la prière traditionnellement. 


\section{La Noix de Cola (ojị)}

La noix de cola qui est produite par les Yorouba dans l'ouest du Nigéria joue des rôles importants chez les Igbo. Il y a des espèces differentes. Certaines noix de cola sont blanches . Il y en a d'autres qui sont vertes ou presque rouges en couleur. Certaines noix comportent deux, quatre, cinq ou meme six lobes. Les noix de cola produites chez les Igbo comportent quatre, cinq ou six lobes. On les présente à une visite pour lui souhaiter la bienvenue. Dans la société igbo la première chose à presenter à des convives pendant la fête est la noix de cola avant de les fêter. La noix de cola exprime l'accueil chalereux.

On raconte l'histoire d'un villageois qui a rendu visite à son cadet dans une grande ville. Son hôte a préparé une sauce de légume au poulet, le favorit de son frère. Il a bu et il a mangé. Mais on ne lui a pas présenté la noix de cola. Au retour au village, il a porté plainte que son frère cadet ne lui a pas bien reçu parce qu'on ne lui a pas offert la noix.

\section{La Craie Blanche (nzu)}

La craie blanche est aussi le symbole d'un accueil chalereux. On peut l'offrir ensemble avec la noix de cola. Mais s'il y a la pénurie de noix de cola la craie blanche est offerte tout seul. Certains font des lignes au nombre de quatre sur le sol pour représenter les quatre jours de la semaine traditionnelle.D'autre part, le rameau de palmier s' emploie dans la rue pour marquer un danger. Si le goudron de la route est cassé ou troué on utilise le rameau pour avertir le chauffeur.

Le rameau de palmier est utilisé aussi pour annoncer un décès. Le rameau attaché sur un véhicule indique qu 'il transporte un cadavre. Le rameau attaché autour d'un étalage indique que le propriétaire a rendu l'âme. 


\section{La Communication Verbale}

La communication verbale comprend l'emploi des organes phonatoires pour exprimer des idées. On produit des sons. Les sons pourraient être des paroles, l'huluement; etc. Dans les paroles on prononce les mots d'une manière que la comprehension est à la portée de tous.

Mais en ce qui concerne l'hululement, bien que les organes phonatoires soient employés les sons produits ne sont pas clairs. La comprehension de l'énoncé ne peut pas être réalisée par tout le monde. Il n'y a que le groupe de pair, c'està -dire ceux qui font partie du groupe qui seront capables de comprendre ce qui est dit car n'est pas bien pronouncé. On essaie d'imiter le produit par certains oiseaux comme l'hibou et le coocoo.

Il y a deux avantages dans ce système verbal. D'abord le locuteur et l'interlocuteur peuvent se communiquer en étant loin de l'un et l'autre. Le son produit peut aller très loin. On utilise ce système pour situer quelqu' un perdu dans la brousse en cherchant du bois. L'huluement est employé lorsque l'interlocuteur et le locuteur ne veulent pas se faire comprendre par les autres.

\section{Les Instruments de Communications Chez Les Igbo}

On emploie les instruments de communication sont employés aussi pour faire la musique et pour la communication. Tantôt la communication est réalisée par le biais de ces instruments sont à la portée de tous tantôt elle ne l'est pas. Nous avons quelques instruments à discuter ici. 


\section{La Flute (OJA)}

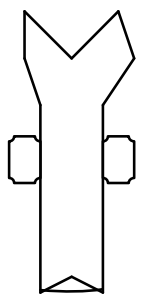

La flute des Igbos est en bois bien taillé. C'est un petit objet dont la longueur ne dépasse pas celle d'un crayon. Elle est caractérisée de quatre trous. On souffle dans le trou situé dans la partie antérieure de l'instrument. On utilize les autres pour changer de ton et de rythme en les bouchant avec les doigts ou en otant ces derniers des trous. La flute est employée pour changer de style dans la dance traditionnelle. De plus la flute traditionnelle est utilisée pour chanter les louanges des gens.

\section{La Corne (OPI)}

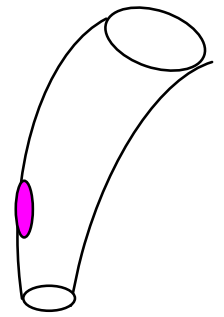

La corne est celle d'une vache ou d'une chèvre vidée et douée de trios ouvertures. L'une se situe à la partie postérieure et l'autre à la partie anterieure. La troisième se trouve au milieu. On souffle dans cette troisième pour produire des sons. On bouche ou debouche les autres ouvertures avec les paumes dans le but de changer de ton ou de rythme. La corne faire la 
musique. Les sons produits par la corne atteignent des regions lointaines mais on exige beaucoup de forces pour souffler.

\section{Le Gong Métallique Gigantesque (UBOM)}
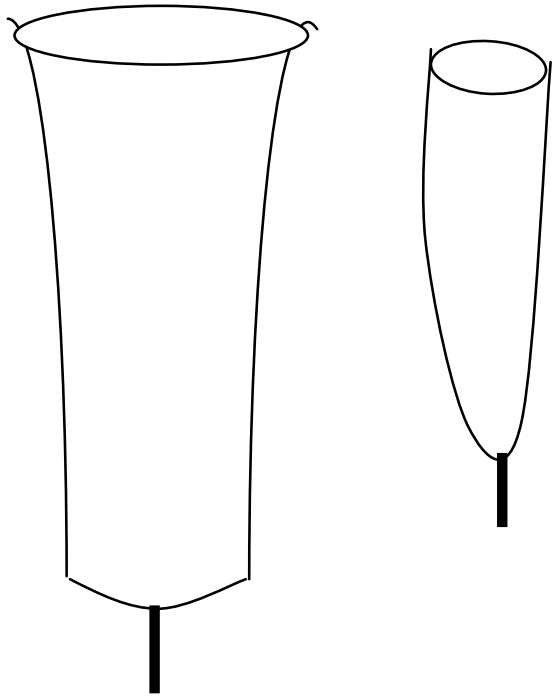

Ce genre de gong métallique produit des sons dans la basse ou basse profonde. Les sons ne couvrent pas une grande distance. On change son en frappant les deux bouts du gong avec une baguette en vue de communiquer ou de changer style de la dance.

\section{Le Gong Métallique a Deux Têtes (OGENE MKPI ABỤỌ)}

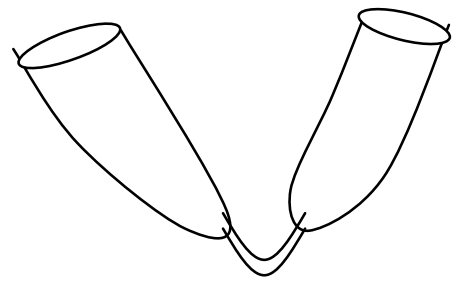


Ce genre de gong est doué de tailles différentes selon l'usage. Les sons produits dependent de la taille du gong. Chaque tête produit des sons différents. On fait la musique, l'annonce et chante le louange de quelqu' un. Pour changer de sons, on frappe les deux bouts antérieur et postérieur avec une baguette.

\section{Le Gong Gigantesque en Bois (IKORO)}

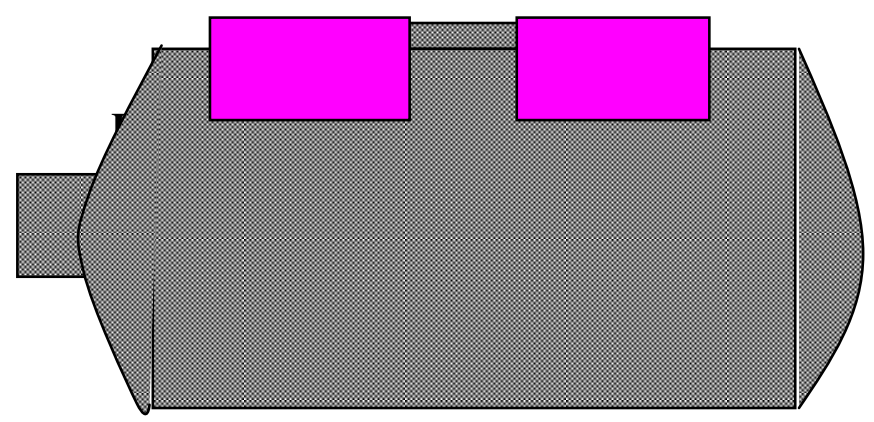

Le gong gigantesque en bois (Ikoro) est un instrument très important chez les Igbo. On frappe l'instrument la nuit pour announcer des grands événements tells que la fête de nouvelle igname, le décès d'une grande personnalité. On considère ikoro comme un instrument sacré utilisé pendant des fêtes, l'enterrement et pour announcer un état d'urgence. Ikoro occupe une position importante dans la direction des communautés traditionnelles. 


\section{Le Tam - Tam (IGBA)}

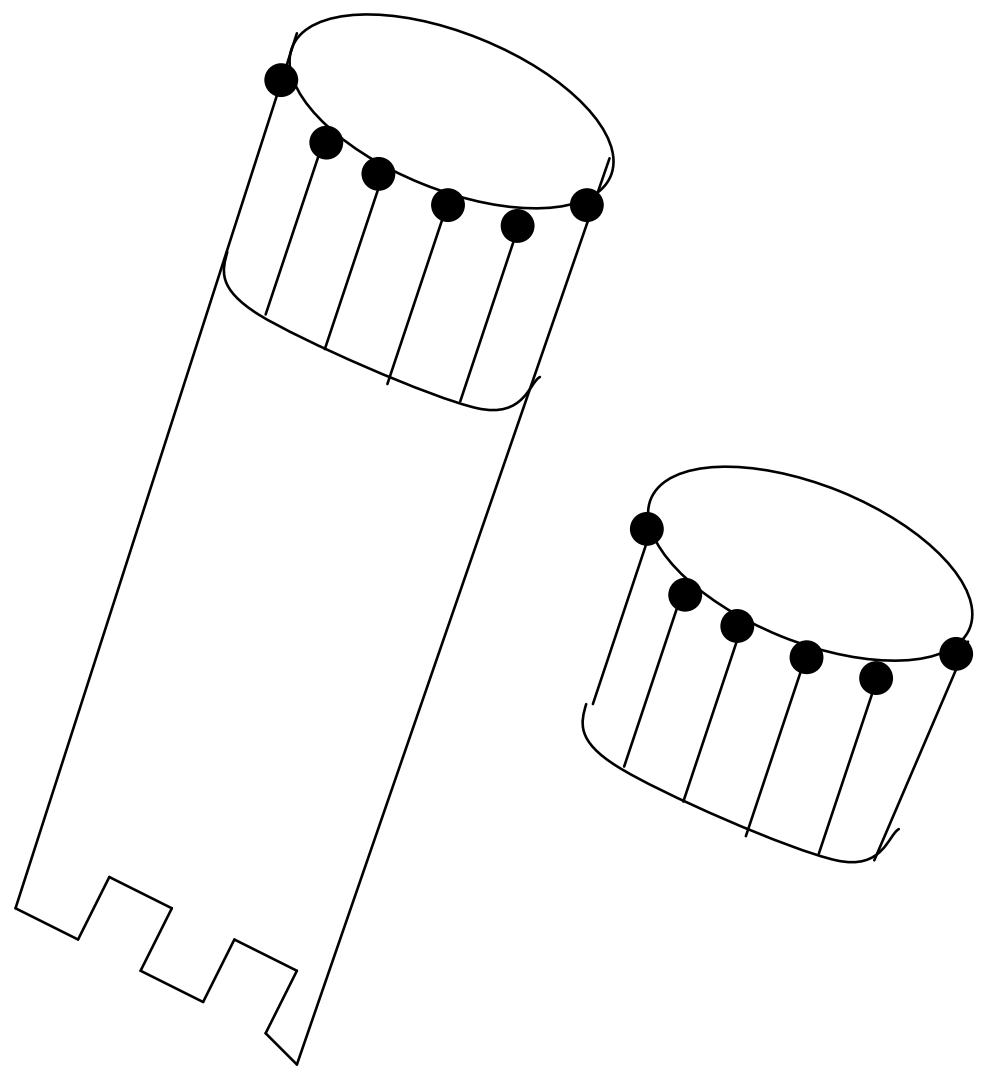

Le tam-tam est de tailles diverses et c'est une sorte de tambour utilisé pour faire la musique et pour transmettre le message. Le tam-tam est formé d'une peau tendue sur un cadre cylindrique 
long ou cour. On fait raisonner le tam-tam à l'aide des baguettes.

\section{Les Proverbes}

Le proverbe est un aspect très important dans la communication chez les Igbo. Selon Achebe dans Things Fall Apart <proverbes are the palm oil with which words are eaten $>$. Version françaises. $<$ Les proverbes sont l'huile de palme avec laquelle on avale les mots. $>$ Pour faciliter la compréhension d'un discours ou pour résumer on utilise les proverbes. L'emploi des proverbes est un art qu'il faut maîtriser pour ne pas les utiliser hors de contexte.

\section{Conclusion}

Les moyens de communication chez les Igbo sont nombreux. On ne peut pas tout citer dans ce travail. Toute fois de nos jours, on constate que certains moyens sont jettés dans l'oubli. On préfère les instruments électroniques. Ce développements menace cet aspect de la culture chez les Igbo. Les gongs et les tam-tams sont peu utilisés dans la transmission d'information. On peut employer les systèmes éléctroniques et les moyens traditionnels ensemble en vue de garder cet aspect de la tradition.

A ce niveau de notre développement on souhaite vivement la coexistence des deux systèmes car < le faucon se perche et le milan se perche aucun ne dira non à son prochain>.

\section{References}

Achebe C. (1957) Things Fall Apart. London :Heinemann.

Robert P. (1985) Le Petit Robert Dictionnaire. Paris : Le Robert - 107, Avenue Parimeilier. 
Maduagwuna S.(2005) Mimes and Gestures for Effective Teaching of Languages in Secondary Schools: a case of French Language.Published in the Book of readings in Language and Literature.Makurdi,CEST.

Agụgụ M. O. (2006) Ndi Igbo na akuko Alaha, Nsukka: EvaUnique Printing and Publishing Company.

Ubesie T. (1978) Odinala ndi igbo Ibada: Oxford University Press.

Samuel Maduagwuna is of the Department of French, Nwafor Orizu College of Education,Nsugbe while Oluchukwu Asadu is of the Department of Modern European Languages, Nnamdi Azikiwe University, Awka. 